\title{
Spectrum Monitoring for Radar Bands using Deep Convolutional Neural Networks
}

\author{
Ahmed Selim*, Francisco Paisana*, Jerome A. Arokkiam, Yi Zhang, Linda Doyle, Luiz A. DaSilva \\ CONNECT center, Trinity College Dublin, Ireland \\ email: $\{$ selimam, paisanaf, a.jerome, zhangy8, linda.doyle, dasilval $\} @$ tcd.ie
}

\begin{abstract}
In this paper, we present a spectrum monitoring framework for the detection of radar signals in spectrum sharing scenarios. The core of our framework is a deep convolutional neural network (CNN) model that enables Measurement Capable Devices (MCDs) to identify the presence of radar signals in the radio spectrum, even when these signals are overlapped with other sources of interference, such as commercial Long-Term Evolution (LTE) and Wireless Local Area Network (WLAN). We collected a large dataset of RF measurements, which include the transmissions of multiple radar pulse waveforms, downlink LTE, WLAN, and thermal noise. We propose a pre-processing data representation that leverages the amplitude and phase shifts of the collected samples. This representation allows our Convolutional Neural Network (CNN) model to achieve a classification accuracy of $99.6 \%$ on our testing dataset. The trained CNN model is then tested under various SNR values, outperforming other models, such as spectrogram-based CNN models.
\end{abstract}

\section{INTRODUCTION}

Spectrum sharing frameworks reliant on Geo-location Databases (GLDBs) tend to leave spectrum underutilized. The reasons behind this are well understood and pointed out in the TV White Space (TVWS) and Citizens Broadband Radio Service (CBRS) literature [1], [2]. GLDBs, in their current form, cannot cope with the mobility of incumbents, and rely on theoretical propagation models that do not efficiently account for factors like terrain elevation and other obstructions [3].

Spectrum monitoring networks combined with Radio Environment Maps (REMs) may prove to be an important tool for addressing the aforementioned issues [1]. As REMs rely on real-time RF measurements, they can provide a more exact picture of the current spectrum occupancy across time, frequency and space. Environment Sensing Capability (ESC), currently under investigation for the CBRS band, is an example of the spectral efficiency gains such an approach can provide [4]. REMs may also allow operators to optimize their resource allocations and identify potential sources of interference, and provide regulators with a tool for spectrum enforcement, without the costs of sending specialized staff to the field for interference analysis [5].

One technical challenge for spectrum monitoring networks is in the selection of sensing algorithms that enable MCDs to infer spectrum occupancy. Several of the existing works on REM assume the use of received signal strength indicator (RSSI) or energy detection-based methods to delimit incumbents' protection zones [1], [6]. This may not, however, be an effective approach when multiple Radio Access Technologies (RATs), with different priorities for spectrum access and levels

\footnotetext{
${ }^{*}$ Denotes equal contribution
}

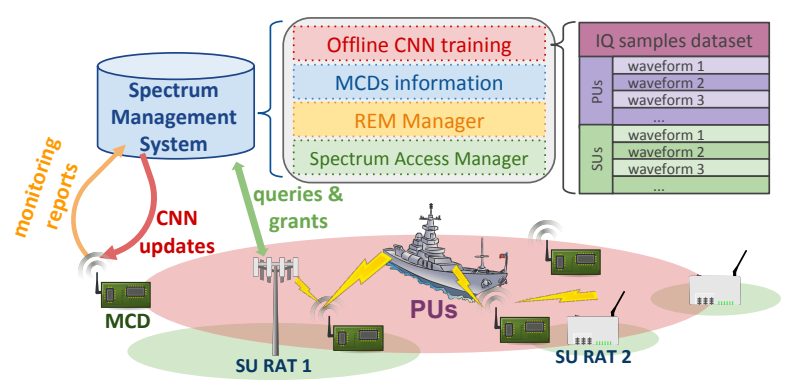

Fig. 1: Illustration of the proposed deep learning framework for spectrum monitoring. MCDs report sensing results using CNN models trained by the spectrum manager.

of protection from interference coexist in the same band. In such scenarios, there is a requirement for MCDs to be able to discriminate different users and technologies, which can only be achieved with more advanced signal classification algorithms.

Over the last decades, expert feature search and cyclostationary detection algorithms have dominated cognitive radio approaches on signal detection and classification [7]-[10]. However, the design of these specialized solutions has been proven to be time-demanding and inefficient, especially in license-exempt bands and some military-controlled bands, where a large number of signal types may coexist. With recent breakthroughs in machine learning, more flexible approaches based on neural networks started outperforming expert systems at specific tasks [11]. However, these solutions have been primarily focused on the specific problem of modulation recognition (ModRec), which only represents a sub-task of the emitter classification problem in spectrum monitoring. In contrast to ModRec, in this paper, we consider a sharing scenario where RATs can employ adaptive modulation schemes, the incumbents and Secondary Users (SUs) may follow incompatible channelization strategies, and overlapping between their signals might occur. One such scenario is spectrum sharing in radar bands, which is being considered by regulators and the wireless communication industry [4], [12], [13].

In this paper, we assess the applicability of CNNs for spectrum monitoring, in particular, in the identification of radar signals. Our proposed framework, illustrated in Figure 1. consists of a spectrum management system that handles: (i) the channel access authorization of SUs, (ii) REM generation based on MCDs' sensing reports, (iii) and training of the CNN models that the MCDs will employ to identify incumbent signals. This CNN training procedure takes place offline, using 
datasets with all possible signal types, including incumbents and SUs, that may access the spectrum. One advantage of our proposed framework is being software-based. In particular, the MCDs' CNN models can be updated whenever new RATs are introduced in the targeted frequency band. Another advantage is obfuscation, as MCDs do not need explicit information about the radar systems' waveforms, which meets the secrecy requirements of some military bands.

We considered a radio environment where three types of technology may coexist: radars as incumbents, and WLAN and commercial downlink LTE as SUs. The main goal of this work is to build a machine learning-based signal classification model to successfully identify the presence of radar signals. To do this, we started by collecting and labeling a large dataset of RF measurements. We then assess the suitability of different signal representations (e.g. spectrograms and amplitude variation) to perform our classification task. Based on this analysis, we propose a unique signal representation that leverages amplitude and phase shift properties of radar signals. This representation allows our proposed CNN model to achieve robust and reliable classification, as will be shown in the results section.

\section{Spectrum Monitoring In RADAR Systems}

In this section, we discuss the main challenges associated to the identification of radar signals, in low SNRs or when overlapped with other communication signals. In particular, we briefly characterize different features of radar pulses, and what makes them distinguishable in comparison to signals of current commercial broadband RATs such as WLAN and LTE. This effort will provide some intuition about what data representation should be utilized by $\mathrm{CNN}$ models.

\section{A. Radar Signal Model}

To construct our system model, we imagine a single MCD at a fixed position, receiving signals from several communication systems in its surroundings. A radar system is suddenly turned on and it starts transmitting pulses through a directive antenna. To create an REM of the radar system's coverage, the MCD has to be able to detect its pulses when they are at their highest amplitude. This generally corresponds to the instants of time when the radar antenna main beam directly illuminates the MCD.

The samples received by the MCD from a single radar in an ideal channel can be modeled as follows,

$$
x[n]=\sum_{m=-\infty}^{+\infty} A_{m} \cdot\left(p\left[n-m \Delta t_{m}^{\mathrm{pri}}\right] * h_{m}[n]\right) \cdot e^{2 \pi j n f_{m}^{c} / f_{0}} .
$$

Here, $n$ is the sample index and $m$ is the pulse index. The pulse shape $p[\cdot]$ defines the Intra-Pulse Modulation (IPM) and Pulse Width (PW). The pulse amplitude $(A)$ suffers sharp variations with time due to the radar antenna steering. The pulse centre frequency $f^{c}$ causes a rotating phase shift in the received IQ samples. The Pulse Repetition Interval (PRI) ( $\left.\Delta t^{\mathrm{pri}}\right)$ of the radar defines the interval between pulses' Time of Arrival (TOA). The radio channel $h_{m}[\cdot]$ has the effect of dispersing the pulse shape $p[\cdot]$ over time.

\section{B. Radar Signal Representations}

In Figure 2, we illustrate three examples of commonly used radar IPMs: Pulse Carrier (PC), Linear Frequency Modulation (LFM) and Barker BPSK Phase Modulation (PM). These samples were generated through the radar waveform emulator described in [14] and transmitted over the air. The spectrum analyzer was operating at a sampling rate of $20 \mathrm{MS} / \mathrm{s}$ and misaligned with the transmitter by $3 \mathrm{MHz}$. The first row of Figure 2 shows the IQ and amplitude of different radar pulses over time. The following rows illustrate the phase difference $(\Delta \phi)$ between consecutive IQ samples, spectrogram, and magnitude square of the signal's DFT, respectively.

The radar pulse waveforms are quite recognizable under any of the chosen representations. However, each representation provides a different level of robustness to non-ideal channel and radio front-end effects. Taking for instance Amplitude+IQ subfigures, we can observe that the amplitude envelope of the received pulses is not perfectly constant over time. In the PM phase difference plot, it can also be seen that the phase shift of the BPSK pulse does not ever reach 180 degrees. These phenomena are generally a consequence of multipath or the limited receiver's sampling rate that filters out high frequency components of the pulses. In [15], the authors show that in long distance NLOS outdoor settings, radar pulses can incur even further amplitude and IQ distortions than the ones illustrated in Figure 2

Misalignments between the transmitter and receiver's centre frequencies, on the other hand, have the effect of offsetting the pulse position in the DFT and spectrogram plots, and altering the mean of its $\Delta \phi[n]$ curve. This mean value can be computed as follows,

$$
\begin{aligned}
\Delta \phi & =\measuredangle\left(p[n] \cdot e^{2 \pi j n \frac{f^{c}}{f_{0}}} \cdot\left(p[n-1] \cdot e^{2 \pi j(n-1) \frac{f^{c}}{f_{0}}}\right)^{*}\right) \\
& =\measuredangle\left(p[n] \cdot p^{*}[n-1] \cdot e^{-2 \pi j \frac{f^{c}}{f_{0}}}\right) \\
& =-2 \pi \frac{f^{c}}{f_{0}} \measuredangle\left(p[n] \cdot p^{*}[n-1]\right) .
\end{aligned}
$$

where $\measuredangle$ is the angle operator, and $\measuredangle\left(p[n] \cdot p^{*}[n-1]\right)$ is the $\Delta \phi[n]$ when the transmitter and receivers are exactly frequency aligned. In IQ versus time representations, on the other hand, any frequency misalignment will lead to a significant alteration in the pulse curve shape.

Classification of radar signals based on DFT may provide very poor performances at low SNRs, due to the generally very short duration of radar pulses compared to the DFT window. In addition, frequency selective multipath or the presence of multiple pulses per DFT window will significantly affect the shape of the resulting DFT.

\section{SU Signal Representations}

We selected two types of commercial technologies as secondary users: WLAN and DL-LTE. In Figure 3, we show representative examples of the IQ and amplitude over time, phase difference, spectrogram, and DFTs of these two signals, captured from the commercial bands of $2.462 \mathrm{GHz}$ and 906 $\mathrm{MHz}$, respectively. The spectrum analyzer's sampling rate is 

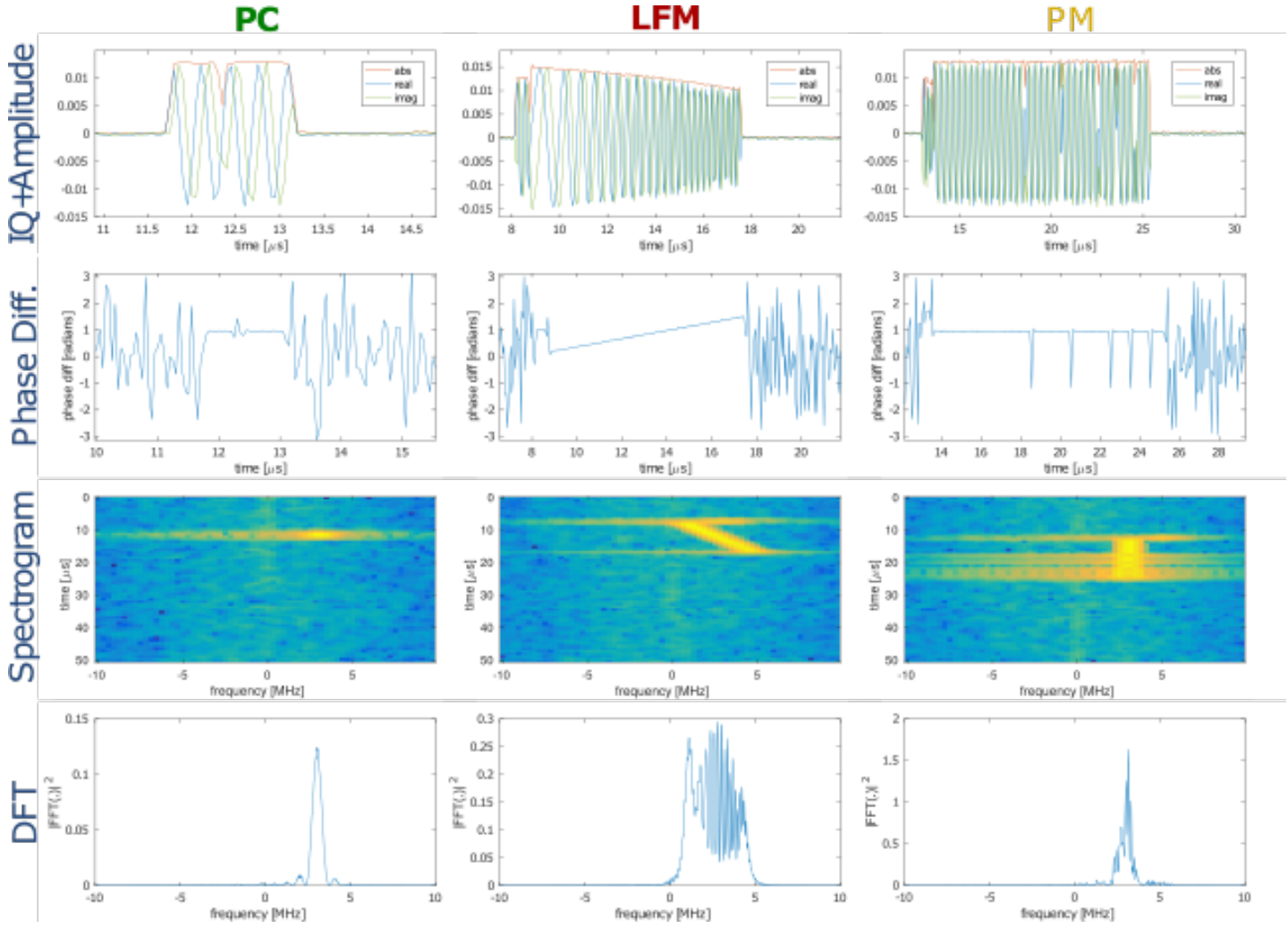

Fig. 2: Examples of possible radar pulse waveforms.

set to $20 \mathrm{MS} / \mathrm{s}$. As illustrated in the WLAN spectrogram, multiple users can contend for access to WLAN bands, with different frequency alignments, and transmissions are expected to occur in a bursty fashion using the CSMA/CA protocol. Although licensed LTE eNB are generally deployed more sparsely and transmit in a continuous manner, their transmit power allocation over time and frequency varies according to the load and scheduler of the cell. The LTE bursty wideband emissions observed in Figure 3 are caused by the LTE reference signaling, and becomes more noticeable the less loaded the cell is. LTE systems also have configurable bandwidth, ranging from $1.4 \mathrm{MHz}$ to $20 \mathrm{MHz}$ without carrier aggregation.

The transmission burstiness and the superposition of misaligned WLAN signals can unpredictably alter the shape of the DFTs, making it an ill-suited representation for WLAN signal classification. On the other hand, both WLAN phase difference and the spectrogram plots show quite distinctive features compared to radar pulses. The WLAN signal amplitude is fairly constant over time and its packets generally display longer durations than radar pulses.

The flexible scheduling schemes of LTE systems lead to an almost infinite number of possible DFT shapes, spectrogram, phase difference, and IQ representations. The short durations of its OFDM symbols may also cause non-constant amplitude bursts of around $66 \mu \mathrm{s}$ of duration, which can be challenging to discriminate from radar pulses, when employing a hand-coded energy-based pulse detection approach. The most distinctive features of LTE's bursts of energy, compared to radar pulses, are their wider instantaneous phase and frequency spreads.

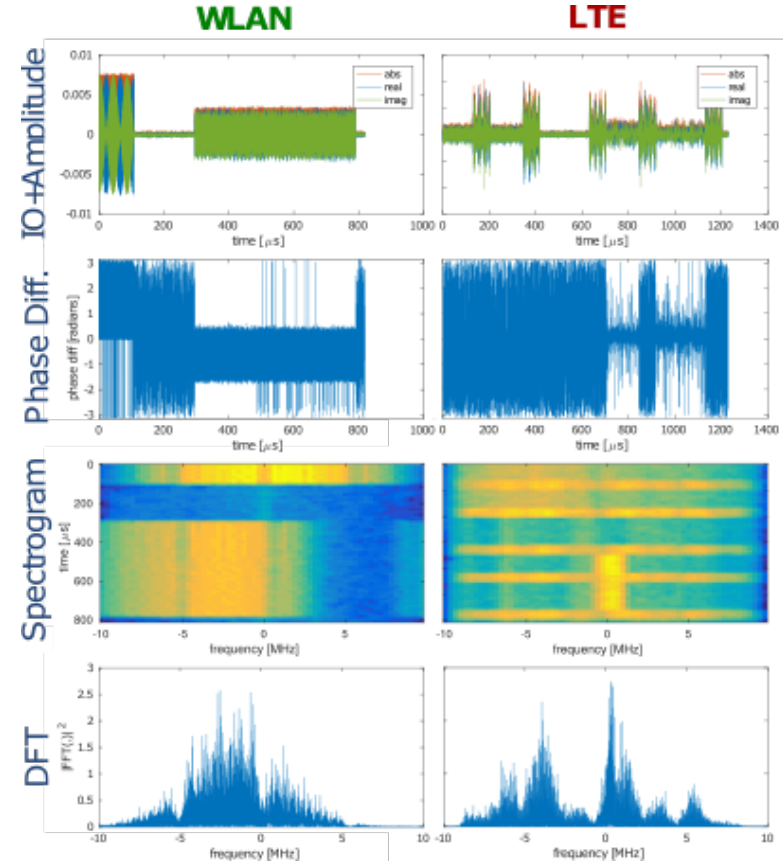

Fig. 3: Illustrations of WLAN and LTE waveforms.

\section{CNN FOR RADAR SIGNAL CLASSIFICATION}

\section{A. Data Collection}

Our data collection setup consisted of two USRP N210 front-ends. One USRP, which we denote as SDR-Radar, is used for generating and transmitting radar pulses every $1 \mathrm{~ms}$. The second USRP, called SDR-MCD, operates as an MCD, 
TABLE I: Set of radar parameters and bands utilized during the $\mathrm{CNN}$ training and testing phases.

\begin{tabular}{|c|c|c|c|c|}
\hline Parameters & \multicolumn{4}{|c|}{ Values } \\
\hline$\Delta f[\mathrm{MHz}]$ & \multicolumn{4}{|c|}{$,-3,0,3,6$} \\
\hline IPM & PC & PC & LFM & PM (Barker 13) \\
\hline PW $[\mu \mathrm{s}]$ & 2 & 10 & 10 & 10 \\
\hline$f^{e}[\mathrm{MHz}]$ & 0 & 0 & 4 & 0 \\
\hline
\end{tabular}

TABLE II: Number of 1024-sized IQ chunks used per scenario

\begin{tabular}{|c|c|c|}
\hline class & training dataset & testing dataset \\
\hline 0: radar signal present & 20000 & 4000 \\
\hline 1: radar signal absent & 20000 & 4000 \\
\hline \hline Total & 40000 & 8000 \\
\hline
\end{tabular}

receiving and storing samples into a file at $20 \mathrm{MS} / \mathrm{s}$. We performed this sample collection over three different bands: LTE $906 \mathrm{MHz}$, ISM $2462 \mathrm{MHz}$, and the $2300 \mathrm{MHz}$ band, where the latter represented a radio medium that is free of interference. To increase our CNN model's robustness to channel and radio front-end effects and increase its capability to generalize to multiple radar waveforms, we altered the parameters of the SDR-Radar in a random fashion. Table II shows the set of parameter configurations used to collect radar signals over different bands. These parameters include the IPM, PW, frequency excursion $f^{e}$, and frequency misalignment $\Delta f$ between the SDR-Radar and SDR-MCD.

We partitioned each collected file into chunks of 1024 IQ samples. Table II shows the total number of chunks used during the training and testing phases. Here, class 0 includes chunks with radar-only, radar+WLAN, and radar+LTE samples. Class 1 includes LTE-only, WLAN-only, and noise. All collected chunks are checked manually and mislabelled data is removed.

\section{B. CNN Models: Design and Performance Evaluation}

Inspired by the aforementioned discussion, as well as the visual characteristics of the radar pulses in Figure 2 and WLAN and LTE signals in Figure 3, we infer that spectrograms, amplitude and phase difference representations are the most suitable for our CNN solution. These representations are not sensitive to frequency misalignments and phase shifts. In addition to this, radar signals have unique characteristics under these transformations, which helps $\mathrm{CNN}$ models to perform reliable classification.

For simplicity, we will start with spectrograms and show how they can be used as data representations. The same framework can be extended to other representations in a straightforward manner. Figure 4 shows our proposed CNN architecture. Here, the input of the network is a spectrogram of size $64 \times 64$. To reach the output of the network, the spectrogram is fed to five consecutive convolutional layers, followed by 2 fully connected layers. The $i$ th convolutional layer consists of $N_{i}$ filters with dimension of $11 \times 11,5 \times 5$, $3 \times 3,3 \times 3,3 \times 3$ for $i=1,2, . ., 5$, respectively. The output of the last fully connected layer is fed to a softmax classifier to compute the probability $P(y=k \mid x ; \theta)$ for $k \in\{0,1\}$, where $x$ denotes the input spectrogram, and $\theta$ denotes model parameters. $k=0$ denotes the presence of radar signals. The CNN model is trained on $40 \mathrm{~K}$ spectrograms, for $25 \mathrm{~K}$ iterations with batch size of 50. The training is performed using the stochastic gradient descent algorithm in which the weights of the network are updated after each iteration to minimize classification errors. The learning rate starts with 0.01 and is divided by a factor of 10 every $5 \mathrm{~K}$ iterations. The momentum is set to 0.9 and the regularization parameter is set to 0.001 to avoid over-fitting. The model is trained using the Caffe framework [16] on a powerful GPU (Tesla K40c). After training, the model is tested over $8 \mathrm{~K}$ spectrograms (test dataset) achieving classification accuracy of $98.6 \%$.

The same CNN model can be used for other data representations with slight modifications to reflect the change in the input size. It is possible to build a CNN model for the amplitude representation (A-CNN), and another model for the phase difference $\Delta \phi(\mathrm{P}-\mathrm{CNN})$. We found that both models achieve worse performance than the spectrogram-based CNN model (S-CNN). However, we also observe that amplitude and phase difference representations are correlated in radar signals (see Fig. 3. first two rows). We therefore combine both representations and feed them as input to our CNN model. The intuition behind this is to allow the CNN model to capture the inter-dependencies between phase difference and amplitude signals present in radar pulses that the ACNN and P-CNN models were not capable of. To achieve this, first, we normalize both representations to have values from 0 to 1 . Amplitude representations are divided by their maximum values, while phase difference representations are divided by $2 \pi$. We then concatenate the two representations in a $1024 \times 2$ matrix. For convenience, we reshape this matrix into $64 \times 64 \times 2$. Now, the same $\mathrm{CNN}$ architecture can be used, however, with a slight variation in the first convolutional layer (filter dimensions are set to $2 \times 11 \times 11$ ). All other network layers and training parameters remain the same. The trained model achieved classification accuracy of $99.6 \%$ on our testing dataset. Although both models can provide high classification accuracies, we observe that the Amplitude+Phase differencebased CNN model (AP-CNN) achieves better performance than the S-CNN model. This is due to the fact that AP-CNN model, unlike S-CNN, utilizes phase information.

\section{EXPERIMENTAL RESULTS}

\section{A. Discrimination between Radar Signals and SUs}

Let $P_{S}, P_{A P}$ denote the probability $P\left(y=0 \mid x ; \theta_{S}\right)$ and $P\left(y=0 \mid x ; \theta_{A P}\right)$, where $\theta_{S}, \theta_{A P}$ denote model parameters of the S-CNN and the AP-CNN models, respectively. Figures 5 and 6 show few examples from our testing dataset and the generated CNN results. The first row of each section represents 3 spectrograms, while their corresponding AP-representations are shown in the second row. The numbers below the spectrograms and AP-representations indicate the obtained $P_{S}$ and $P_{A P}$ values, respectively. Here, for visualization purposes, we show AP-representations as RGB images. Normalized amplitude is assigned to the red channel, normalized phase difference is assigned to the green channel and the blue 

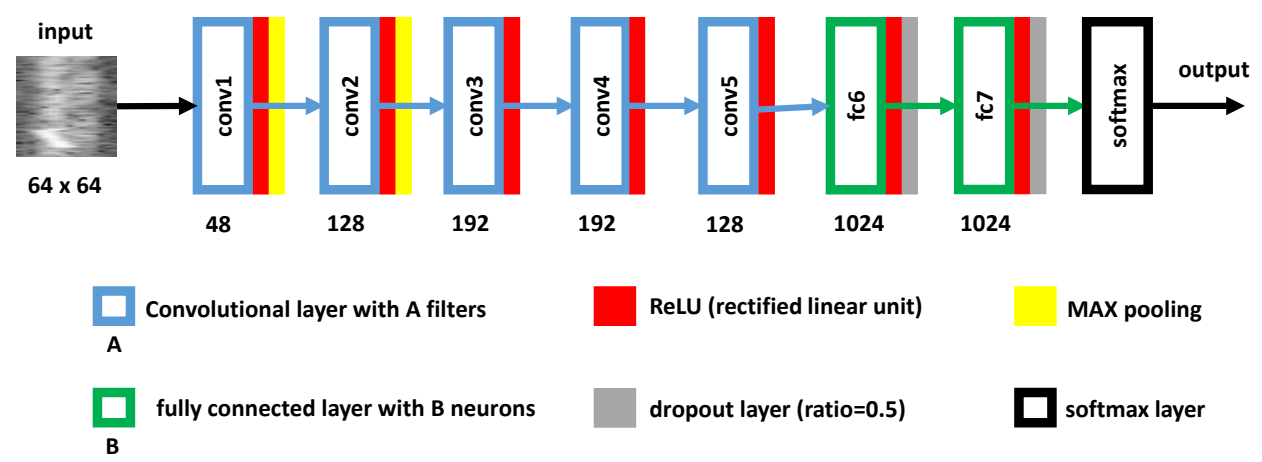

Fig. 4: Proposed CNN architecture.

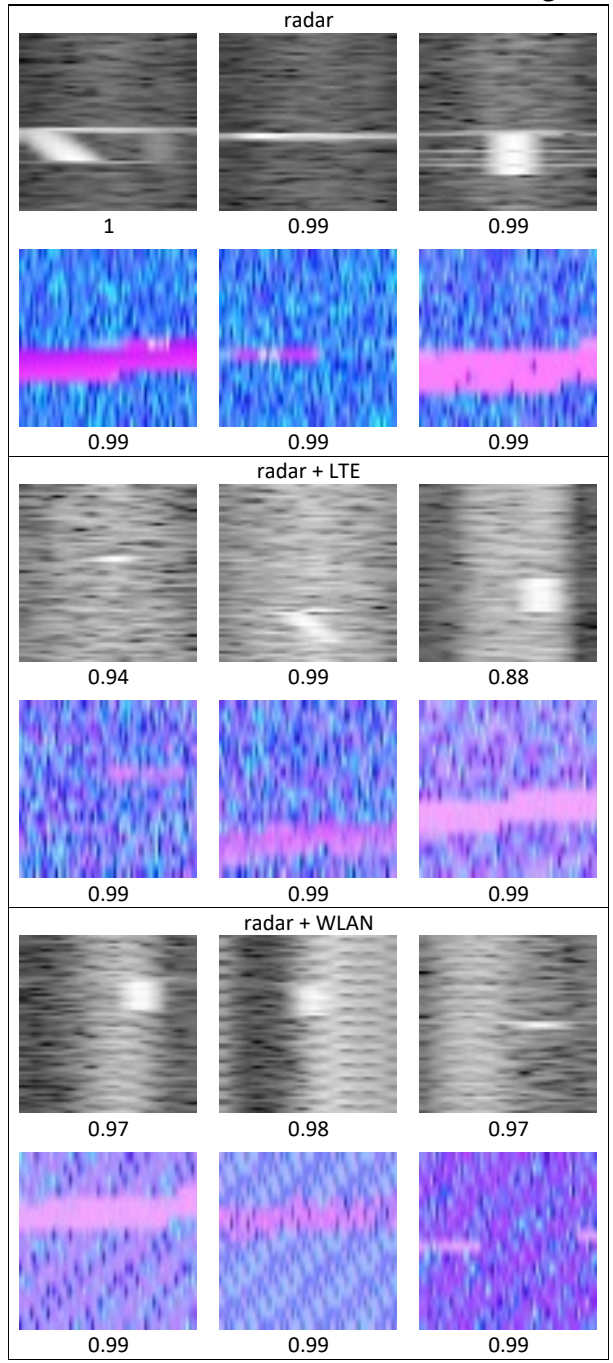

Fig. 5: Examples from our testing dataset when radar signals are present. $P_{S}$ and $P_{A P}$ are shown below spectrograms and AP representations, respectively.

channel is set to 1 . We observe that both CNN models successfully identified the presence/absence of radar signals in the tested cases.

\section{B. Sensitivity to Noise}

In this section, we test the detection performance of both S-CNN and AP-CNN models under different noise levels. For

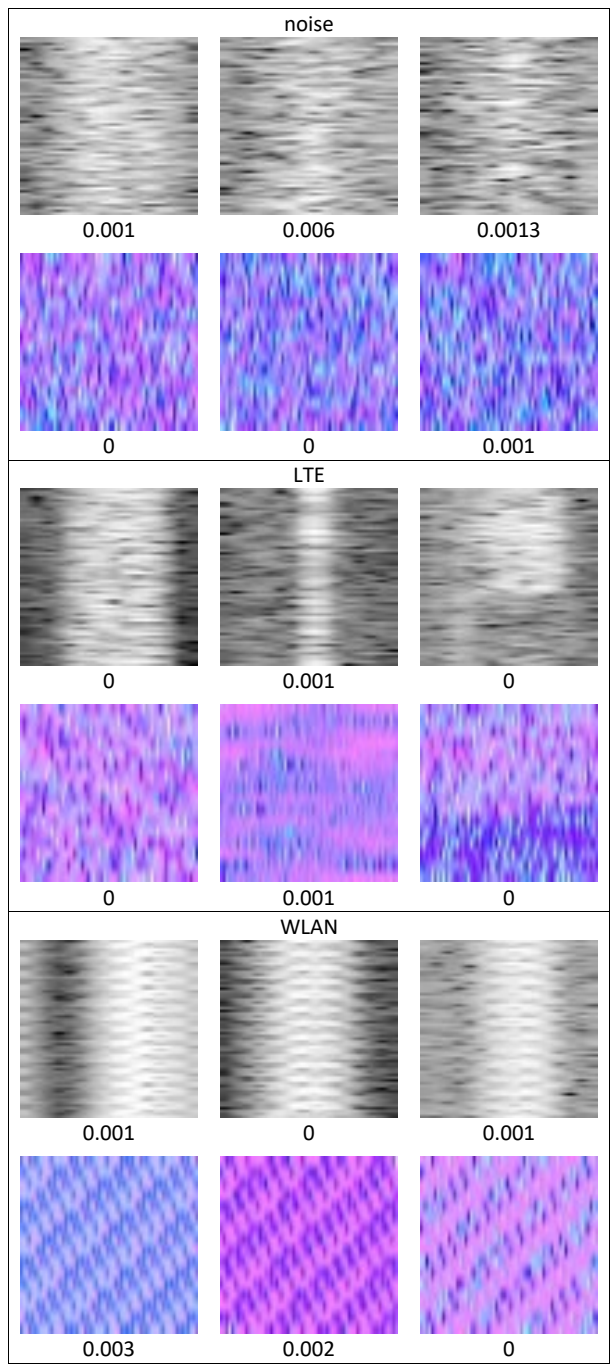

Fig. 6: Examples from our testing dataset when radar signals are absent. $P_{S}$ and $P_{A P}$ are shown below its associated spectrograms and AP representations, respectively.

this test, our dataset consists of 18 sets, each containing 670 IQ chunks of radar+noise samples. Each set was collected using different SDR-Radar USRP N210 transmit gains. The transmitted waveforms were the same as the ones shown in Table I. To estimate the average peak SNR (PSNR) of each set of chunks, we computed the mean power of the radar pulses' samples and the mean power of the remaining samples within 


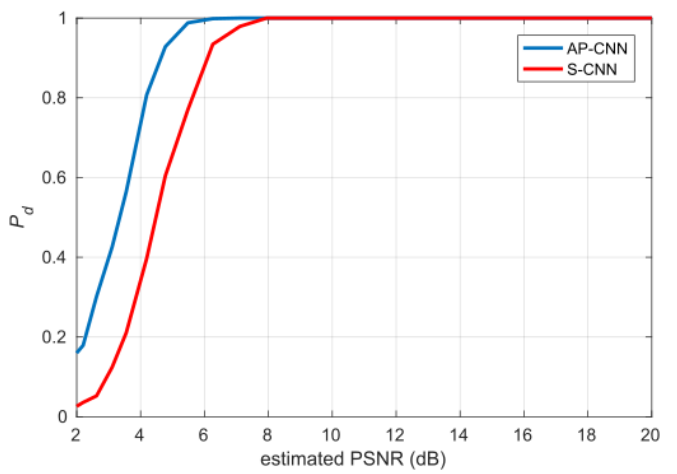

Fig. 7: Probability of pulse detection for the LFM waveform at different PSNR levels.

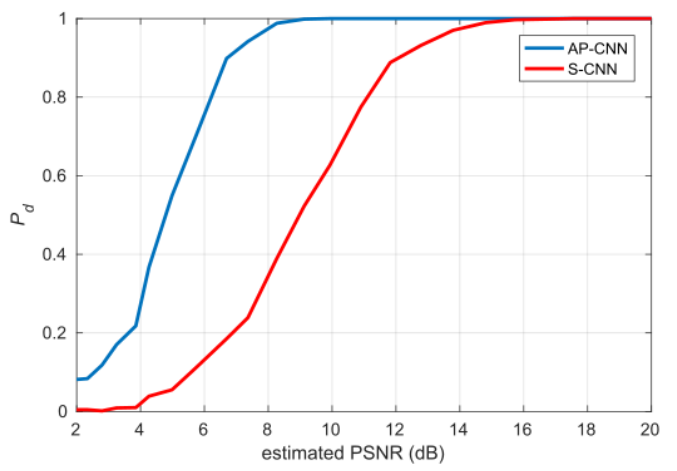

Fig. 8: Probability of pulse detection for the PC waveform with a duration of $2 \mu \mathrm{s}$ at different PSNR levels.

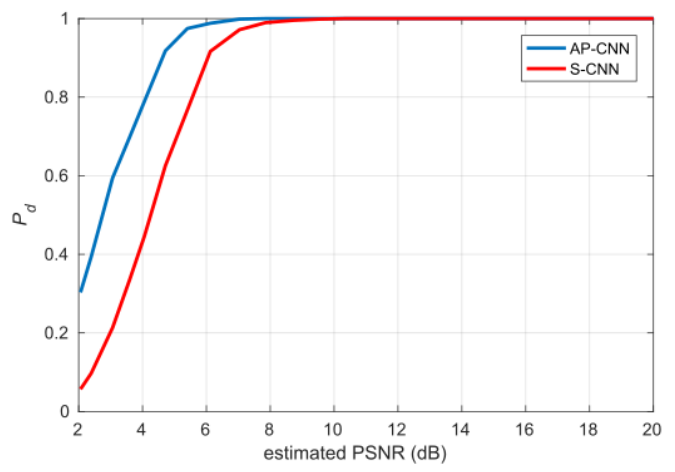

Fig. 9: Probability of pulse detection for the PC waveform with a duration of $10 \mu$ s at different PSNR levels.

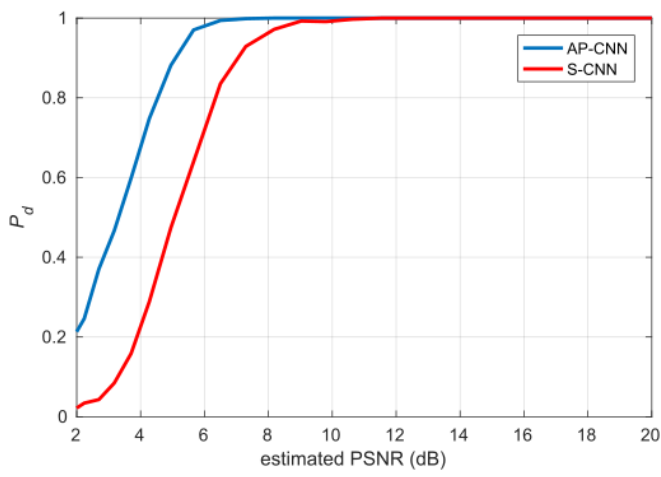

Fig. 10: Probability of pulse detection for the PM waveform at different PSNR levels. each set. $P_{d}$ is the detection probability of radar pulses.

Figures 7, 8, 9 and 10 show the obtained results using $\mathrm{S}-\mathrm{CNN}$ and AP-CNN models. It is clear that the AP-CNN outperforms the S-CNN model, especially at low PSNR values, and for short pulse durations. The main reason behind this success is the fact that the phase difference information embedded in radar pulses helps the CNN model to distinguish them from the general impulsiveness of thermal noise.

\section{CONClusion And Future Work}

In this work, we presented a spectrum monitoring framework for radar bands. Our goal was to detect the presence of radar signals even in the case of simultaneous transmission of LTE and WLAN systems. This is achieved through deep CNNs. We tested the performance for different data representations and concluded that our proposed Amplitude+Phase Difference representation enables CNNs models to obtain high classification accuracy and it is more robust to noise.

As future work, we intend to extend our dataset to include other RATs, such as LAA-LTE, Multefire, and NB-IoT as SUs, and more radar waveforms. Our training and testing datasets will be available online for benchmarking and further research. Another direction for this work is to utilize our new trained CNN models in wireless systems' control and decision making such as the work presented in [17].

\section{ACKNOWLEDGMENT}

This publication has emanated from the research supported by the European Commission Horizon 2020 Program under grant agreements no. 688116 (eWINE) and no. 732174 (ORCA), and co-funded under the European Regional Development Fund from Science Foundation Ireland under Grant Number 13/RC/2077 (CONNECT). Deep learning models were trained on the machines maintained by the Trinity Center for High Performance Computing.

\section{REFERENCES}

[1] L. Gavrilovska, J. Van De Beek, Y. Xie, E. Lidström, J. Riihijärvi, P. Mähönen, V. Atanasovski, D. Denkovski, and V. Rakovic, "Enabling LTE in TVWS with radio environment maps: From an architecture design towards a system level prototype," Computer Communications, vol. 53, pp. 62-72, 2014.

[2] FCC, Report \& Order and Second Further Notice of Proposed Rulemaking (FNPRM) FCC 15-47, GN Docket No. 12-354, 2015.

[3] F. Paisana, N. Marchetti, and L. A. DaSilva, "Radar, TV and Cellular Bands: Which Spectrum Access Techniques for Which Bands?" pp. 124, 2014.

[4] E. Drocella, J. Richards, R. Sole, F. Najmy, A. Lundy, and P. Mckenna, "3.5 GHz Exclusion Zone Analyses and Methodology," Tech. Rep., 2015.

[5] A. Nika, Z. Zhang, X. Zhou, B. Y. Zhao, and H. Zheng, "Towards commoditized real-time spectrum monitoring," 1st ACM MobiCom Workshop on Hot Topics in Wireless (HotWireless), pp. 25-29, 2014.

[6] X. Ying, S. Roy, and R. Poovendran, "Incentivizing crowdsourcing for radio environment mapping with statistical interpolation," in IEEE International Symposium on Dynamic Spectrum Access Networks (DySPAN), 2015, pp. 365-374.

[7] A. Swami and B. M. Sadler, "Hierarchical digital modulation classification using cumulants," IEEE Transactions on communications, vol. 48, no. 3, pp. 416-429, 2000. 
[8] K. Kim, I. A. Akbar, K. K. Bae, J.-S. Um, C. M. Spooner, and J. H. Reed, "Cyclostationary approaches to signal detection and classification in cognitive radio," in New frontiers in dynamic spectrum access networks, 2007. DySPAN 2007. 2nd IEEE international symposium on. IEEE, 2007, pp. 212-215.

[9] F. Paisana, N. Prasad, A. Rodrigues, and R. Prasad, "An alternative implementation of a cyclostationary detector," in 15th International Symposium on Wireless Personal Multimedia Communications (WPMC), 2012, pp. 45-49.

[10] B. Ramkumar, "Automatic modulation classification for cognitive radios using cyclic feature detection," IEEE Circuits and Systems Magazine, vol. 9, no. 2, 2009.

[11] T. J. OShea, J. Corgan, and T. C. Clancy, "Convolutional radio modulation recognition networks," in International Conference on Engineering Applications of Neural Networks, 2016, pp. 213-226.

[12] ITU-R Recommendations M.1652-1, "Dynamic frequency selection in wireless access systems including radio local area networks for the purpose of protecting the radiodetermination service in the $5 \mathrm{GHz}$ band," vol. $1,2011$.

[13] FCC, "Enabling Innovative Small Cell Use In 3.5 GHZ Band," Washington, Notice of Proposed Rulemaking (NPRM) \& Order Docket 12-148, 2012.

[14] J. F. Santos, F. Paisana, N. J. Kaminski, J. M. Marquez-Barja, and L. A. DaSilva, "Context-aware radar modeling framework," in IEEE International Symposium on Dynamic Spectrum Access Networks (DySPAN), 2015, pp. 113-122.

[15] F. Paisana, L. A. Dasilva, and N. Marchetti, "Spectrum Sharing Techniques for Radar Bands," PhD Thesis, Trinity College, Dublin, 2017.

[16] Y. Jia, E. Shelhamer, J. Donahue, S. Karayev, J. Long, R. Girshick, S. Guadarrama, and T. Darrell, "Caffe: Convolutional architecture for fast feature embedding," arXiv preprint arXiv:1408.5093, 2014

[17] F. Paisana, A. Selim, M. Kist, P. Alvarez, J. Tallon, C. Bluemm, A. Puschmann, and L. Dasilva, "Context-Aware Cognitive Radio Using Deep Learning," in IEEE International Symposium on Dynamic Spectrum Access Networks (DYSPAN), Spectrum Challenge, 2017. 